\title{
Multifocal Colonic Wall Abscesses during Anti-Tumor Necrosis Factor (TNF)- $\alpha$ Therapy for a Patient with Ulcerative Colitis: A Very Rare Manifestation of Infectious Complications
}

\author{
Toshiro Fukui, Mio Takahashi, Takashi Okazaki, Takashi Tomiyama, \\ Norimasa Fukata, Yugo Ando and Kazuichi Okazaki
}

\begin{abstract}
A 24-year-old woman was transferred to our hospital under suspicion of an exacerbation of her known ulcerative colitis. Colonoscopy revealed an edematous swelling and multifocal discharge of pus throughout the descending colon, concurrent with active ulcerative colitis findings in the rectum and sigmoid colon. Computed tomography showed a thickened wall and multifocal abscesses within the wall of the descending colon. Two weeks after starting antimicrobial therapy, she was discharged home. This is the first case report of multifocal colonic wall abscesses. In order not to increase the risk of serious infection associated with anti-TNF$\alpha$ therapy, proper qualification and strict monitoring are essential.
\end{abstract}

Key words: ulcerative colitis, biologics, adverse drug event, abscess

(Intern Med 56: 1157-1161, 2017)

(DOI: 10.2169/internalmedicine.56.8041)

\section{Introduction}

Tumor necrosis factor (TNF)- $\alpha$ is one of the most important proinflammatory cytokines, playing key roles in the host defense system against infection. However, it has been implicated in the pathogenesis of many inflammatory and autoimmune diseases, including inflammatory bowel disease (IBD) (1). The reduction of TNF- $\alpha$ levels by anti-TNF- $\alpha$ antibodies leads to diminished pathologic inflammatory responses in these diseases (1).

Currently in Japan, there are four basic categories of medications used in the treatment of IBD: aminosalicylates [5-aminosalicylic acid (5-ASA) and salazosulfapyridine], corticosteroids, immunomodulators (azathioprine, 6-mercaptopurine, cyclosporin, and tacrolimus), and biologic therapy (anti-TNF- $\alpha$ ) (2). The high clinical efficacy of anti-TNF- $\alpha$ therapy has resulted in its wider application for both Crohn's disease (CD) and ulcerative colitis (UC) (3). The number of anti-TNF- $\alpha$-treated patients is increasing annually in Japan. The two most frequently used monoclonal anti-
TNF- $\alpha$ antibodies approved for IBD are infliximab (IFX) and adalimumab (ADM).

The safety profile and drug-related adverse events (AEs) are very important issues associated with every new therapy. Although anti-TNF- $\alpha$ therapy is generally considered to be safe, one of the major risks is the small but significant risk of serious infection (4). Corticosteroids, immunomodulators, and anti-TNF- $\alpha$ therapy, especially when used in combination, are associated with an increased risk of infection $(4,5)$. It must be noted, however, that the risk of infection with combination therapy is comparatively low, and that each case should be evaluated to assess the risks and benefits of treatment options as appropriate, according to the disease activity. Adherence to treatment guidelines can avoid increasing the risk of serious infection associated with antiTNF- $\alpha$ therapy and thereby lead to a safer course in IBD patients (2).

To our knowledge, this is the first case report of multifocal colonic wall abscesses, which was a complication observed in the course of unsuccessful treatment for $\mathrm{UC}$ with IFX.

The Third Department of Internal Medicine, Division of Gastroenterology and Hepatology, Kansai Medical University, Japan Received for publication July 22, 2016; Accepted for publication September 25, 2016

Correspondence to Dr. Toshiro Fukui, fukuitos@hirakata.kmu.ac.jp 

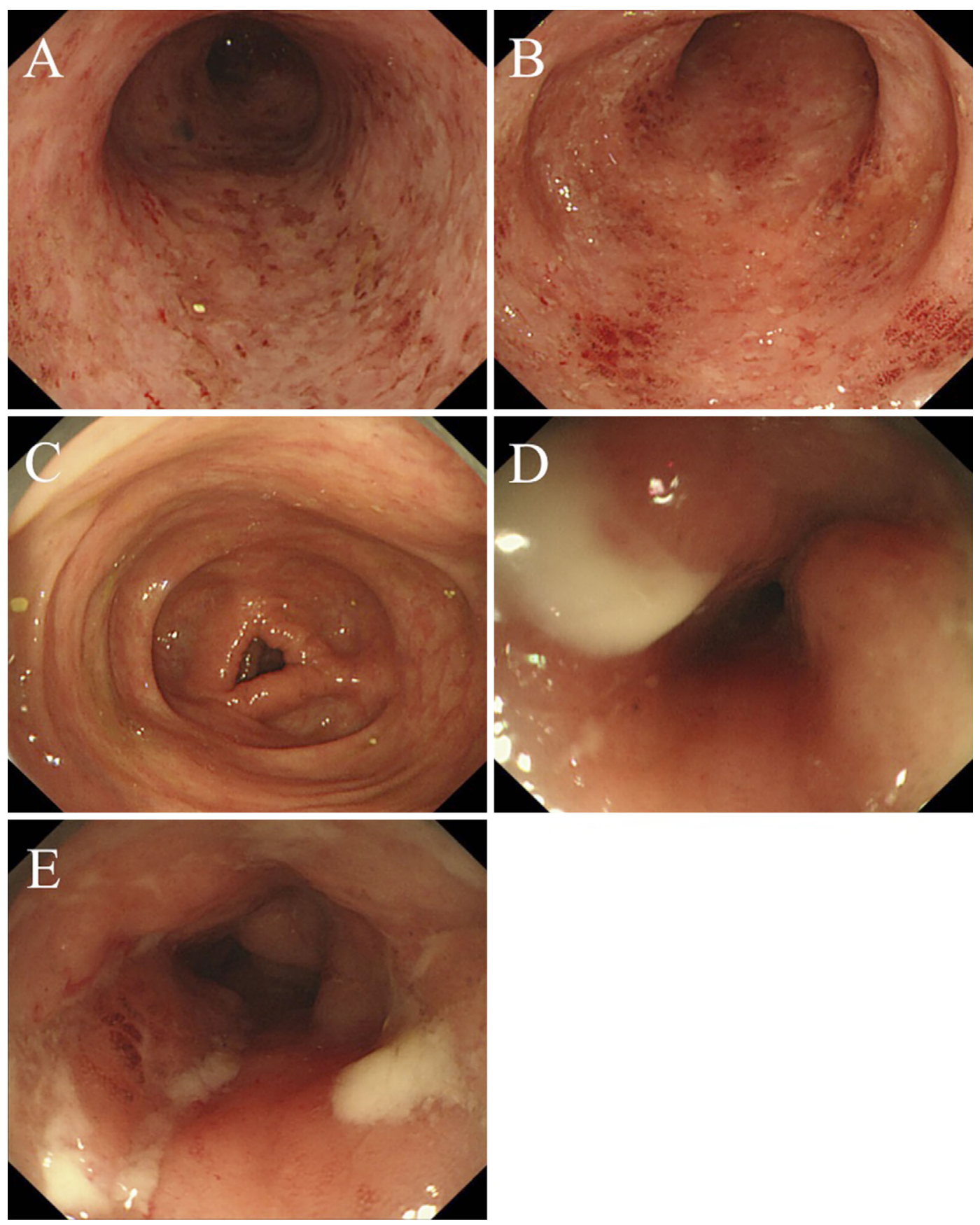

Figure 1. Colonoscopic images in the rectum (A), sigmoid colon (B), transverse colon (C), and descending colon (D, E) on admission. Colonoscopy revealed the exacerbation of ulcerative colitis in the rectum (A) and sigmoid colon $(B)$. Although there were no active findings from the cecum to the transverse colon $(\mathrm{C})$, we found long luminal edematous swelling and multifocal massive discharge of pus throughout the descending colon (D, E). The mucosa of the descending colon appeared to be nearly intact.

\section{Case Report}

A 24-year-old woman with UC diagnosed 2 years previously was transferred to our hospital due to a 1-week history of a high-grade fever, bloody diarrhea, frequent bowel movements, and severe pain on the left side of the abdomen. Her UC was not well controlled with an oral 5-ASA therapy, so she was started on IFX in July 2014. She had al- ready received the induction doses as well as 8 maintenance doses at $5 \mathrm{mg} / \mathrm{kg}$, and she had maintained clinical remission for about 1.5 years. The final administration was 8 weeks prior to the start of the above-mentioned symptoms. She had neither history of travel nor any recent exposure with sick individuals, and her medical history was unremarkable except for UC. On a physical examination, the patient was febrile $\left(39.5^{\circ} \mathrm{C}\right)$ with mild tachycardia, and an abdominal examination revealed moderate tenderness with hyperactive 

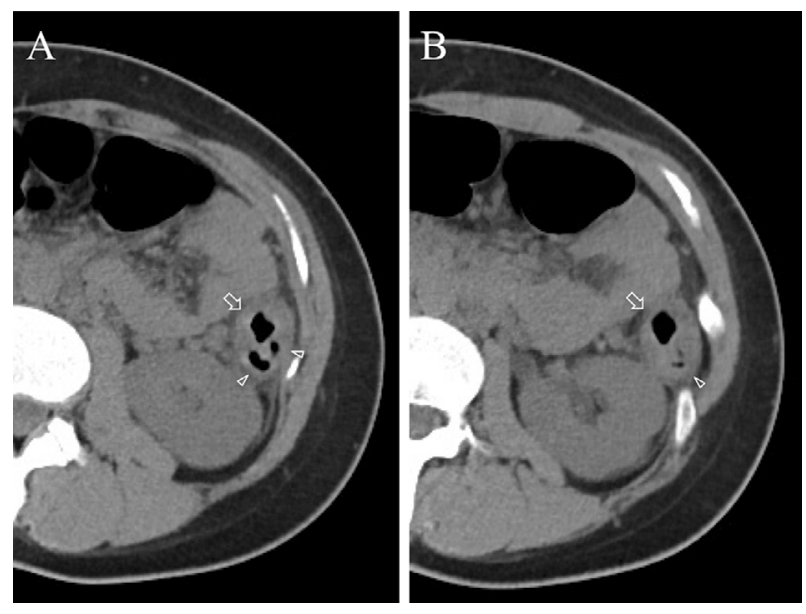

Figure 2. A computed tomography scan immediately after the colonoscopy $(\mathrm{A}, \mathrm{B})$. The scan showed a diffusely thickened wall with a narrowed lumen and intramural air-filled abscess cavities and multifocal low-density areas of abscesses within the wall of the descending colon. The arrows and arrowheads indicate the lumen of the descending colon and air-filled abscess cavities, respectively.

bowel sounds and mild rebound tenderness in the left side of the abdomen.

Her laboratory examination revealed a decreased albumin of $3.4(\mathrm{~g} / \mathrm{dL})$, elevated C-reactive protein of $8.53(\mathrm{mg} / \mathrm{dL})$, elevated erythrocyte sedimentation rate of $47.1(\mathrm{~mm})$, elevated white blood cell count of $124\left(10^{2} / \mu \mathrm{L}\right)$, with $81.0 \%$ neutrophils (normal range: 42-77\%), hemoglobin within normal limits at $13.0(\mathrm{~g} / \mathrm{dL})$, blood platelet count within normal limits at $29.9\left(10^{4} / \mu \mathrm{L}\right)$, negativity for hepatitis B virus antigen and antibody, negativity for hepatitis $\mathrm{C}$ virus antibody, negativity for chlamydia trachomatis antibody, negativity for syphilis, $\beta$-D-glucan within normal limits at $<6.0(\mathrm{pg} / \mathrm{mL})$, negativity for tuberculosis bacterium specific interferon $\gamma$, and negativity for cytomegalovirus antigen. Polymerase chain reaction analyses were negative for both cytomegalovirus and tuberculosis bacterium genes in the colon lesions. No significant pathogens, including bacteria, fungi, viruses, and parasites, were isolated from her blood or were found in the contents of her colon. She tested negative for HIV and human T-lymphotropic virus type (HTLV)-I viruses.

Colonoscopy on admission revealed widespread shallow ulcers in the rectum and coarse mucosa with mucous, pus, and blood in the sigmoid colon (Fig. 1A and B). The mucosal vascular pattern disappeared, and the erythematous mucosa was friable and bled easily. Although there were no active UC findings from the cecum (terminal ileum) to the splenic flexure (Fig. 1C), we found long luminal edematous swelling and multifocal discharge of pus throughout the descending colon (Fig. 1D and E). The mucosa of the descending colon appeared to be nearly intact. Computed tomography immediately after colonoscopy showed a diffusely thickened wall with a narrowed lumen and intramural air- filled abscess cavities and multifocal low-density areas of abscesses (Fig. 2), particularly within the wall of the descending colon. There were no diverticula of the colon, which was confirmed by the prior findings of colonoscopy and computed tomography. Antimicrobial therapy with doripenem hydrate $0.5 \mathrm{~g}$ intravenously every $8 \mathrm{~h}$ was started, as well as intravenous hyperalimentation. 2 weeks after starting treatment, she became afebrile and pain-free, and her C-reactive protein level was normalized. Oral intake was restarted with an elemental diet, and she was later discharged home after confirmation of the improvement of her condition via colonoscopy (Fig. 3A and B) and computed tomography (Fig. 3C). She is still being followed up at our out-patient department, and both oral and anal administration of 5-ASA have maintained clinical remission of her UC. IFX has not been reintroduced.

\section{Discussion}

Anti-TNF- $\alpha$ therapy has revolutionized the treatment of chronic inflammatory diseases, especially in cases refractory to conventional treatment modalities. It offers significant benefits in disease control and improving the quality of life. IBD is a lifelong chronic disorder associated with significant morbidity. While anti-TNF- $\alpha$ therapy has also improved the symptoms and long-term outcomes for many patients with IBD, the side effects are not negligible. A well-balanced safety profile is one of the most important elements influencing the acceptance of a therapy. The safety profile of anti-TNF- $\alpha$ therapy seems to be acceptable, especially considering its relevantly high clinical efficacy $(6,7)$. However, patients must be advised about the risk of infections, malignancies, and various other potential AEs with anti-TNF- $\alpha$ therapy before beginning treatment.

Patients with IBD are at an increased risk of developing infections, which can be characterized as non-therapy dependent, i.e. due to IBD, or therapy-dependent, i.e. due to the medications used to treat IBD (8). In a study by Lees et al., which included 620 patient-years of follow-up, the incidence of infections in IBD patients receiving anti-TNF- $\alpha$ treatment was $9.9 \%$ (9). The authors showed that the majority of infectious AEs occurred among patients receiving concomitant immunomodulatory drugs. It must be noted that the majority of patients were on corticosteroids and/or immunomodulators while starting anti-TNF- $\alpha$ treatment, so it is hard to definitively assess whether or not this fact influenced the infectious complication risk.

De novo infections and the reactivation of latent infectious agents are complex processes and represent an increasingly relevant clinical health problem. With the use of antiTNF- $\alpha$ therapy in IBD, the reactivation of latent bacterial and viral infections may occur. In particular, there is increasing awareness regarding latent virus reactivation, such as hepatitis B virus and cytomegalovirus. Screening for relevant viral and bacterial infections is necessary before beginning anti-TNF- $\alpha$ therapy. 

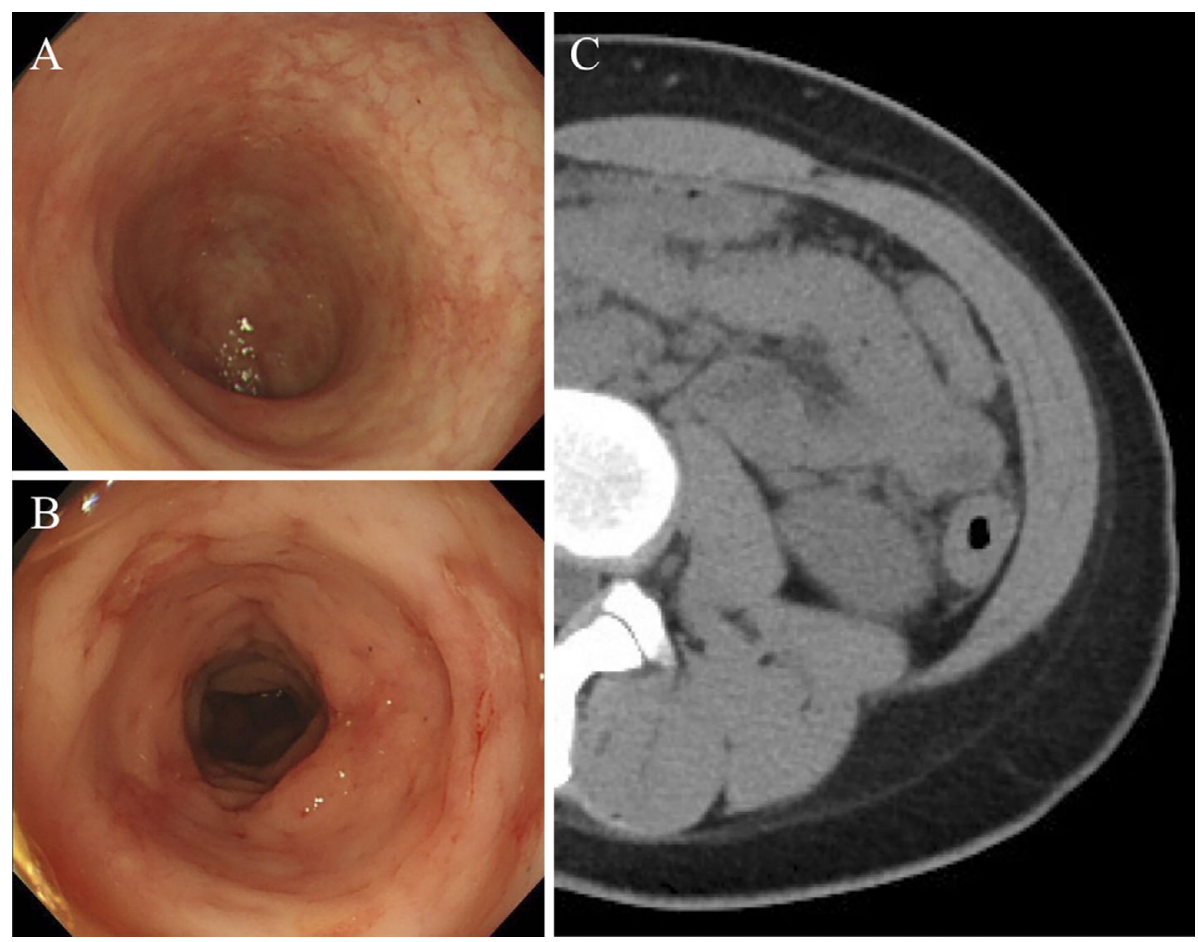

Figure 3. Colonoscopic images in the sigmoid colon (A) and descending colon (B) and a computed tomography scan (C) after improvement. Colonoscopy revealed mucosal healing in the sigmoid colon (A), and the blood vessels became visible. Although the descending colon was in the process of healing (B), we detected the improvement of the mucosal edema, discharge of pus, and the degree of stenosis. Although a computed tomography scan still showed a thickened wall with a narrowed lumen, the intramural air-filled abscess cavities within the wall of the descending colon had diminished.

Determining the long-term outcomes of latent viral reactivation and bacterial infections with anti-TNF- $\alpha$ therapy is essential for clinical decision-making and patient counseling. The optimization, individualization, strict monitoring, and proper qualification of anti-TNF- $\alpha$ therapy can improve the therapeutic outcomes and decrease the risk of AEs. However, since the observation time of the safety profile of antiTNF- $\alpha$ therapy is still short, further assessment is needed in this respect, especially from the perspective of long-term therapy for IBD.

Anti-TNF- $\alpha$ therapy in CD has been well established, and it is currently part of the standard care regimen in most institutions. However, with UC, things get complicated. Unlike $\mathrm{CD}$, we can ultimately resort to colectomy in cases of UC (10). Severe and refractory UC patients may be exposed to the risk and expense of anti-TNF- $\alpha$ therapy while perhaps only delaying subsequent colectomy. It would be a good policy to balance the benefits versus the risks in IBD patients, especially when dealing with cases of UC. Physicians treating patients with anti-TNF- $\alpha$ therapy should therefore be aware of the possibility of such patients developing serious infections.

The lesion in the descending colon of this case had several similar characteristics to phlegmonous colitis (PC) (11-13). PC is an acute suppurative infection caused by bacteria and a rare but often fatal condition. PC preferentially occurs in the cecum to the ascending colon in patients with liver diseases (13). Macroscopically, there is extensive edema in the colon wall, which is also observable by computed tomography. However, there are no macro-abscesses in principle. Microscopically, PC is characterized by diffusely purulent inflammation and disseminated microabscesses in the submucosa, and the mucosa and serosa are usually intact. The areas of mucosal injury in our case were thought to be secondary in nature; elsewhere in the descending colon, the mucosa appeared intact. Some case studies have also suggested that immunosuppression and the consumption of raw seafood are additional risk factors for PC (13). Furthermore, there have been no reports of PC, to our knowledge, associated with $\mathrm{UC}$ or $\mathrm{CD}$. In the present case, immunosuppression due to anti-TNF- $\alpha$ therapy likely played a major role in accelerating the infectious inflammation. We hypothesize that PC may progress toward multifocal colonic wall abscesses, as in this case. In that sense, this case should be diagnosed as "phlegmonous colitis with multifocal wall abscesses" or "phlegmonous colonic wall abscesses." As a local complication of IBD, abscesses are more common in $\mathrm{CD}$ than in UC. Although colonoscopy on admission showed the characteristic findings of UC in the rectum and sigmoid colon, we cannot completely exclude CD or IBD-unclassified at this time. As we have no sufficiently large specimen to evaluate the case in detail and cannot confirm the pathological findings of the colonic wall abscesses, we are limited in our ability to discuss this case. 
Therefore, we must continue a strict follow-up of this patient going forward.

In conclusion, this is the first case report of multifocal colonic wall abscesses during treatment for UC. To ensure prompt and most appropriate treatment, the proper qualification of each therapy and strict monitoring are essential.

The authors state that they have no Conflict of Interest (COI).

\section{References}

1. Brennan FM, Maini RN, Feldmann M. TNF alpha--a pivotal role in rheumatoid arthritis? Br J Rheumatol 31: 293-298, 1992.

2. Matsuoka K, Hibi T. Treatment guidelines in inflammatory bowel disease: the Japanese perspectives. Dig Dis 31: 363-367, 2013.

3. Amiot A, Peyrin-Biroulet L. Current, new and future biological agents on the horizon for the treatment of inflammatory bowel diseases. Therap Adv Gastroenterol 8: 66-82, 2015.

4. Koo S, Marty FM, Baden LR. Infectious complications associated with immunomodulating biologic agents. Infect Dis Clin North Am 24: 285-306, 2010.

5. Toruner M, Loftus EV Jr, Harmsen WS, et al. Risk factors for opportunistic infections in patients with inflammatory bowel disease. Gastroenterology 134: 929-936, 2008.

6. Sousa P, Allez M. Complications of biologics in inflammatory bowel disease. Curr Opin Gastroenterol 31: 296-302, 2015.
7. Stallmach A, Hagel S, Bruns T. Adverse effects of biologics used for treating IBD. Best Pract Res Clin Gastroenterol 24: 167-182, 2010.

8. Epple HJ. Therapy- and non-therapy-dependent infectious complications in inflammatory bowel disease. Dig Dis 27: 555-559, 2009.

9. Lees CW, Ali AI, Thompson AI, et al. The safety profile of antitumour necrosis factor therapy in inflammatory bowel disease in clinical practice: analysis of 620 patient-years follow-up. Aliment Pharmacol Ther 29: 286-297, 2009.

10. Becker JM, Stucchi AF. Treatment of choice for acute severe steroid-refractory ulcerative colitis is colectomy. Inflamm Bowel Dis 15: 146-149, 2009.

11. Yamada T, Sasatomi E, Sato T, et al. Phlegmonous colitis in a patient with liver cirrhosis, hepatocellular carcinoma and acute promyelocytic leukemia. Intern Med 34: 542-545, 1995.

12. Holzer T, Gervaz P, Spahr L, et al. Phlegmonous colitis: another source of sepsis in cirrhotic patients? BMC Gastroenterol 9: 94, 2009.

13. Pang SH, de Silva K, Napoli J, et al. A fatal case of phlegmonous colitis in chronic hepatitis $\mathrm{C}$ receiving combination interferon and ribavirin therapy. Intern Med J 39: 481-484, 2009.

The Internal Medicine is an Open Access article distributed under the Creative Commons Attribution-NonCommercial-NoDerivatives 4.0 International License. To view the details of this license, please visit (https://creativecommons.org/licenses/ by-nc-nd/4.0/).

(C) 2017 The Japanese Society of Internal Medicine http://www.naika.or.jp/imonline/index.html 